\title{
Selecting appropriate methods of knowledge synthesis to inform biodiversity policy
}

\author{
Andrew Pullin ${ }^{1}$ Geoff Frampton ${ }^{2} \cdot$ Rob Jongman $^{3}$ • \\ Christian Kohl $^{4}$ - Barbara Livoreil ${ }^{5}$ - Alexandra Lux ${ }^{6,7}$. \\ György Pataki $^{8} \cdot$ Gillian Petrokofsky $^{9} \cdot$ Aranka Podhora $^{10}$. \\ Heli Saarikoski $^{11} \cdot$ Luis Santamaria $^{12}$ - Stefan Schindler ${ }^{13,14}$. \\ Isabel Sousa-Pinto $^{15} \cdot$ Marie Vandewalle ${ }^{16} \cdot$ Heidi Wittmer $^{17}$
}

Received: 25 January 2016/Revised: 3 May 2016/ Accepted: 7 May 2016/

Published online: 24 May 2016

(C) The Author(s) 2016. This article is published with open access at Springerlink.com

\begin{abstract}
Responding to different questions generated by biodiversity and ecosystem services policy or management requires different forms of knowledge (e.g. scientific, experiential) and knowledge synthesis. Additionally, synthesis methods need to be appropriate to policy context (e.g. question types, budget, timeframe, output type, required scientific rigour). In this paper we present a range of different methods that could potentially be used to conduct a knowledge synthesis in response to questions arising from knowledge needs of decision makers on biodiversity and ecosystem services policy and
\end{abstract}

Communicated by David Hawksworth.

This is part of the special issue on Networking Biodiversity Knowledge.

Andrew Pullin

a.s.pullin@bangor.ac.uk

1 Centre for Evidence-Based Conservation, Bangor University, Bangor, Gwynedd LL57 2UW, UK

2 Southampton Health Technology Assessments Centre (SHTAC), Faculty of Medicine, University of Southampton, Southampton, UK

3 Present Address: Jongman Ecology, Wageningen, The Netherlands

4 Institute for Biosafety in Plant Biotechnology, Julius Kühn-Institut, 06484 Quedlinburg, Germany

5 Fondation pour la Recherche sur la Biodiversité, 195 rue Saint Jacques, 75005 Paris, France

6 ISOE -Institute for Social-Ecological Research, Hamburger Allee 45, 60486 Frankfurt am Main, Germany

7 Senckenberg Biodiversity and Climate Research Centre (BiK-F), Senckenberganlage 25, 60325 Frankfurt am Main, Germany

8 Department of Decision Sciences, Corvinus Business School, Corvinus University of Budapest and Environmental Social Science Research Group (ESSRG), Budapest, Hungary

9 Department of Zoology, Biodiversity Institute, University of Oxford, South Parks Road, Oxford OX1 3PS, UK

10 Leibniz Centre for Agricultural Landscape Research, Eberswalder Str. 84, 15374 Müncheberg, Germany 
management. Through a series of workshops attended by natural and social scientists and decision makers we compiled a range of question types, different policy contexts and potential methodological approaches to knowledge synthesis. Methods are derived from both natural and social sciences fields and reflect the range of question and study types that may be relevant for syntheses. Knowledge can be available either in qualitative or quantitative form and in some cases also mixed. All methods have their strengths and weaknesses and we discuss a sample of these to illustrate the need for diversity and importance of appropriate selection. To summarize this collection, we present a table that identifies potential methods matched to different combinations of question types and policy contexts, aimed at assisting teams undertaking knowledge syntheses to select appropriate methods.

Keywords Evidence-based policy · Biodiversity policy · Decision-making · Ecosystem services $\cdot$ Knowledge brokerage $\cdot$ Evidence synthesis $\cdot$ Knowledge transfer

\section{Introduction}

There is an increasing demand from multiple policy sectors of society for the process of policy making to be informed by the best available knowledge. Knowledge is generated and communicated in diverse formats and its volume is increasing rapidly, presenting significant challenges in searching, collating and synthesising relevant information in a form that is credible, reliable and legitimate from a decision maker's perspective (Cash et al. 2003; Sarkki et al. 2013). This is the case also with knowledge on biodiversity and ecosystem services (and their interactions with other sectors, interests or needs). Management of biodiversity and ecosystem services generates a broad spectrum of knowledge from traditional or indigenous knowledge to experimental and science-based understanding. In turn, the knowledge needs of decision makers reflect this spectrum, and methods of knowledge gathering and synthesis are dependent on the types of uncertainty faced and the social context in which the decision needs to be made (e.g. Breckon and Dodson 2016).

The diversity of approaches to knowledge synthesis is manifest in the diversity of questions generated by the policy and broader decision-making communities (practice and management) (e.g. Sutherland et al. 2006). The challenge is to provide up-to-date synthesis for decision makers, both directly concerned with biodiversity and ecosystem services as

11 Environmental Policy Centre, Finnish Environment Institute, Mechelininkatu 34a, Helsinki, Finland

12 Doñana Biological Station (EBD-CSIC), C/Americo Vespucio s/n, 41092 Seville, Isla de la Cartuja, Spain

13 Division of Conservation Biology, Vegetation and Landscape Ecology, University of Vienna, Rennweg 14, 1030 Vienna, Austria

14 Department of Biodiversity and Nature Conservation, Austria \& Environment Agency Austria, Spittelauer Lände 5, 1090 Vienna, Austria

15 Interdisciplinary Centre for Marine and Environmental Research (Ciimar) and Department of Biology, Faculty of Sciences, University of Porto, R. do Campo Alegre s/n, Porto, Portugal

16 Department of Conservation Biology, UFZ -Helmholtz-Centre for Environmental Research, Permoserstrasse 15, 04318 Leipzig, Germany

17 Department of Environmental Politics, UFZ -Helmholtz-Centre for Environmental Research, Permoserstrasse 15, 04318 Leipzig, Germany 
well as from other sectors that might affect or enter into conflict with biodiversity conservation or with ensuring ecosystem services. The synthesis should be precisely tailored to the request (see Livoreil et al. 2016), in accordance with the policy agenda (which often requires limited time windows and budgets), ensuring its legitimacy from both policymaker and wider stakeholder perspectives. An important challenge is to ensure that concerns from different sectors are adequately taken into account and an agreement on a common knowledge base is reached to avoid having stakeholders with opposing interests presenting opposing evidence or knowledge. This challenge, which applies to the policy process in general, is particularly demanding for environmental and biodiversity policyowing to the inter-disciplinary and complex nature of the opinions and interests involved (Nesshöver et al. 2016).

Synthesis methodologies are ex-ante assessments that do not generate any new empirical data but seek to identify, compile and combine relevant knowledge from various sources, so that it is understandable as a single unit and readily available for decision makers who want to draw on the best available evidence. Methods of synthesis vary according to the type of question, the type of knowledge sought and the policy context (e.g. stage of the policy cycle and timeframe). Selection of an appropriate method can be crucial to the inclusion of appropriate knowledge in the decision-making process. Frustration over knowledge flow can occur both from the policy community, when knowledge is not provided in the necessary time window for decision making, and from the scientific community, when knowledge syntheses are apparently ignored or seen as irrelevant to current evidence needs (Dietz and Stern 1998; Owens 2005; Sharman and Holmes 2010; Jordan and Russel 2014).

In this paper, we aim to present an initial illustrative decision matrix tailored for biodiversity and ecosystem service knowledge that can be developed through future iterations. The matrix's objective is to provide guidance on the selection of appropriate methods of synthesis for a diversity of questions that may be posed by policy makers and, as far as we are aware, is the first effort of this kind applied to environmental knowledge synthesis.

\section{Methods}

A Workshop on 'method selection for providing evidence to policy on biodiversity and ecosystem services' was convened to develop the decision matrix in Frankfurt, Germany in January 2014 (hereafter referred to as the Frankfurt Workshop); participants were invited who had expertise in knowledge synthesis methods from both social and natural sciences in the field of biodiversity and ecosystem services. Participants included six academic researchers from the KNEU project and ten external researchers. All participants were selected for their expertise in policy-relevant research and/or knowledge synthesis methodology (see Table 3 in Appendix 1 for a full list of participants and their affiliations). The workshop considered a range of question types (see below) with respect to knowledge needs to inform decision making arising from three previous workshops convened by the KNEU project in different regions of Europe (hereafter referred to as regional workshops) (Carmen et al. 2015). In these initial regional workshops a broad range of policy makers, stakeholders and scientists had been asked to formulate questions and specify knowledge needs broadly following the methods of Sutherland et al. (2006) involving initial collection of questions followed by discussion and prioritisation sessions. The outcomes then 
informed the Frankfurt Workshop in terms of identifying the spectrum of potential requests submitted to a knowledge synthesis process.

Questions arising from the three regional workshops were classified by the Frankfurt Workshop participants into different types with regard to the evidence sought, as follows:

I. Seeking better understanding of an issue (including predictions and forecasting):

1. Seeking greater understanding or predictive power (e.g. how does green infrastructure contribute to human well-being?)

2. Scenario building to analyse future events (e.g. how will the risk of flooding change under different climate change scenarios?)

3. Horizon scanning (e.g. what will be the most significant novel threats to biodiversity in 2050?)

4. Seeking understanding of changes in time and space (e.g. how has the distribution and abundance of rabies in fox populations changed in the last 10 years?)

II. Identifying appropriate ways and means of realising certain decisions

5. Seeking measures of anthropogenic impact (e.g. what is the impact of wind farm installations on bird populations?)

6. Seeking measures of the effectiveness of interventions (e.g. how effective are marine protected areas at enhancing commercial fish populations?)

7. Seeking appropriate methodologies and associated trade-offs (e.g. what is the most reliable method for monitoring changes in carbon stocks in forest ecosystems?)

8. Seeking optimal management (e.g. what is the optimal grazing regime for maximizing plant diversity in upland meadows?)

III. Improving understanding of possibilities and boundaries for decision-making:

9. Assessing public opinion and perception (e.g. is there public support for badger culling in the UK?)

10. Seeking people's understanding and providing definitions (e.g. how do different people or groups understand ecosystem services?)

At the Frankfurt Workshop, participants were asked to identify and describe, based on their experience, possible policy contexts in which any question may arise and to characterize these in terms of the constraints they might imply for the choice of synthesis method. Through a series of breakout sessions the workshop participants were subsequently asked to consider the suitability of different knowledge synthesis methodologies for each type of question, for each policy context identified. Candidate methodologies were contributed by the workshop participants during the workshop and therefore represent the collective experience of those assembled and are not a comprehensive list. Some methodologies were not included because their purpose is not primarily knowledge synthesis (see "Discussion and conclusion" section).

For the purpose of selecting suitable methodologies a prior (theoretical) assumption was made at the workshop that an existing synthesis would not be available to the decision maker and a new synthesis would therefore have to be conducted and a methodology selected. Methodologies differ widely in their 'robustness' as measured by their transparency (the extent to which all actions and decisions can be reported), rigour (the effort expended to minimise error in the findings), repeatability (the extent to which the method can be repeated by a third party) and susceptibility to bias (the extent to which the methodology addresses and reduces potential for bias in the findings) (Gough et al. 2012). In constructing the matrix, participants selected more robust methodologies in policy 
scenarios where these characteristics become more important (e.g. 'high risk of serious consequences if wrong conclusion is reached'). The most rigorous methodologies were always selected when the policy context allowed.

Workshop participants mapped methods against types of questions and against contextual factors to indicate how well methods are suited to inform each type of question and how well they are expected to perform in the different contexts. Participants subsequently used this process to specify the most promising methods for each combination of question type and policy-context based on the participants' expertise and knowledge of the synthesis methods. A table was drafted during the workshop and modified through subsequent discussion among the authors of this paper (all but three of whom were workshop participants) that could be directly used as a decision support tool for anyone considering the commissioning or conduct of a knowledge synthesis.

\section{Results}

The policy contexts identified in the Frankfurt Workshop and described in the following list are not exhaustive but they are examples of factors that might influence choice of synthesis methodology. We recognise that they are potentially overlapping and interrelated:

\section{Time constraints}

The timeframe over which policy decisions need to be made (the policy window) can sometimes be very short (days to weeks). This places limits on the knowledge synthesis that can be achieved or encourages forms of synthesis that can be conducted and updated rapidly.

\section{Financial resource constraints}

Alongside time constraints there are always financial constraints and knowledge-synthesis methods may be confined to low cost options.

\section{Controversy caused by conflicts of evidence}

Knowledge needs may arise in relation to a disagreement over the interpretation and implications of the current evidence, or its robustness in terms of the variability of existing results. This may require transparent, rigorous, independently conducted (by actors perceived by stakeholders in the conflict to have no vested interest in the outcome) and inclusive synthesis methods that minimise susceptibility to bias by engaging key actors in designing research questions and discussing conclusions on the basis of evidence.

\section{Controversy caused by conflicts of values and/or interests}

Knowledge needs may differ according to vested interests (legitimate or otherwise) in the outcome and/or a fundamental difference in values and beliefs on the part of two or more groups. This may require substantial stakeholder engagement to generate an acceptable question (or questions) as well as transparent, rigorous and inclusive synthesis methods that provide reliable evidence regarded as legitimate by the parties involved. 


\section{Serious and/or unacceptable consequences of making the wrong decision}

Where the consequences of making a wrong decision are regarded as a high risk to a decision maker they may require transparent, rigorous and independently conducted synthesis methods that minimise susceptibility to bias and/or clearly state what the potential biases are, thus providing a clear audit trail to justify the decision.

\section{Diversity of knowledge and information}

Where the question demands the synthesis of a high diversity of different types of knowledge and/or many different perspectives need to be included, it may require interand transdisciplinary methods and approaches that are able to structure diversity, identify commonalities and differences, or rank alternatives.

\section{High uncertainty}

In situations where there is significant uncertainty or variability in knowledge, methods may be required that seek to examine sources of uncertainty and variability in results, and synthesise knowledge taking such uncertainty and/or variability into account. Such methods would provide a best estimate of the truth together with statements of confidence in that estimate.

The identified methodologies are drawn from the natural and social sciences and all have been applied to some extent to support decision making in environmental and other related sectors. Definitions are provided in Table 1 together with explanations of their suitability to explain why they might be chosen for a particular combination of question and policy context.

Table 2 presents an example matrix of methodologies that might be suitable for different question types in different policy contexts. It is not exhaustive and represents the initiation of what could be a more extensive effort to provide guidance in this area. We detected that for many combinations of questions and policy constraints there is more than one possible method. The matrix suggests appropriate methods according to the most prominent constraint characterizing a particular decision setting. For some of these, e.g. very restricted time available, this limits the possible choice of method to expert consultation or causal chain analysis for practically all types of knowledge needs (first row). In the case of controversy of evidence, the choice of appropriate method depends much more on the type of knowledge need (third row). In practice, it is likely that several of these characteristics or constraints will apply in any given situation so that further elaboration of the context (e.g. the nature of evidence sought, qualitative, quantitative and/or the estimated amount of evidence available) would be necessary to make a final decision. For example, in the context of controversy or conflicts of evidence, there may also be secondary contexts, such as time and resource constraints, that would shift the balance of choice toward more rapid methods.

The selection of synthesis methods will have to consider the requirements and constraints of each method highlighted in Table 1 above in relation to the policy context. For example, while systematic review is a very robust knowledge synthesis methodology and can provide highly transparent and reliable results, for many specific knowledge requests there will not be sufficient evidence available to justify a systematic review. Constraints such as insufficient or disputed evidence (e.g. determined by a 'quick scoping' of the literature, Defra 2015) might in many cases make it necessary to resort to joint fact finding or double-sided critique while time and resource constraints and short policy window could justify expert consultation or focus groups. 


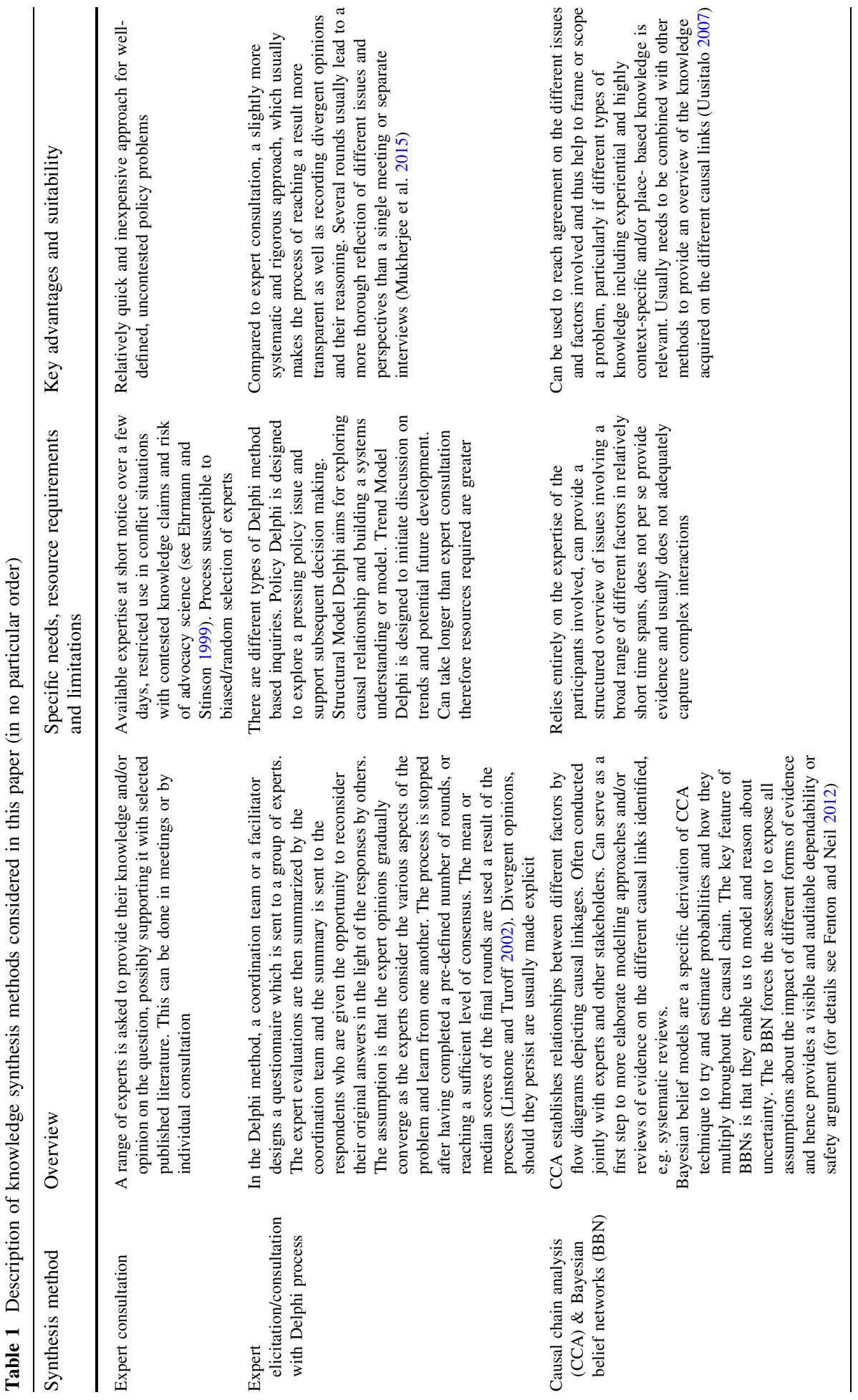




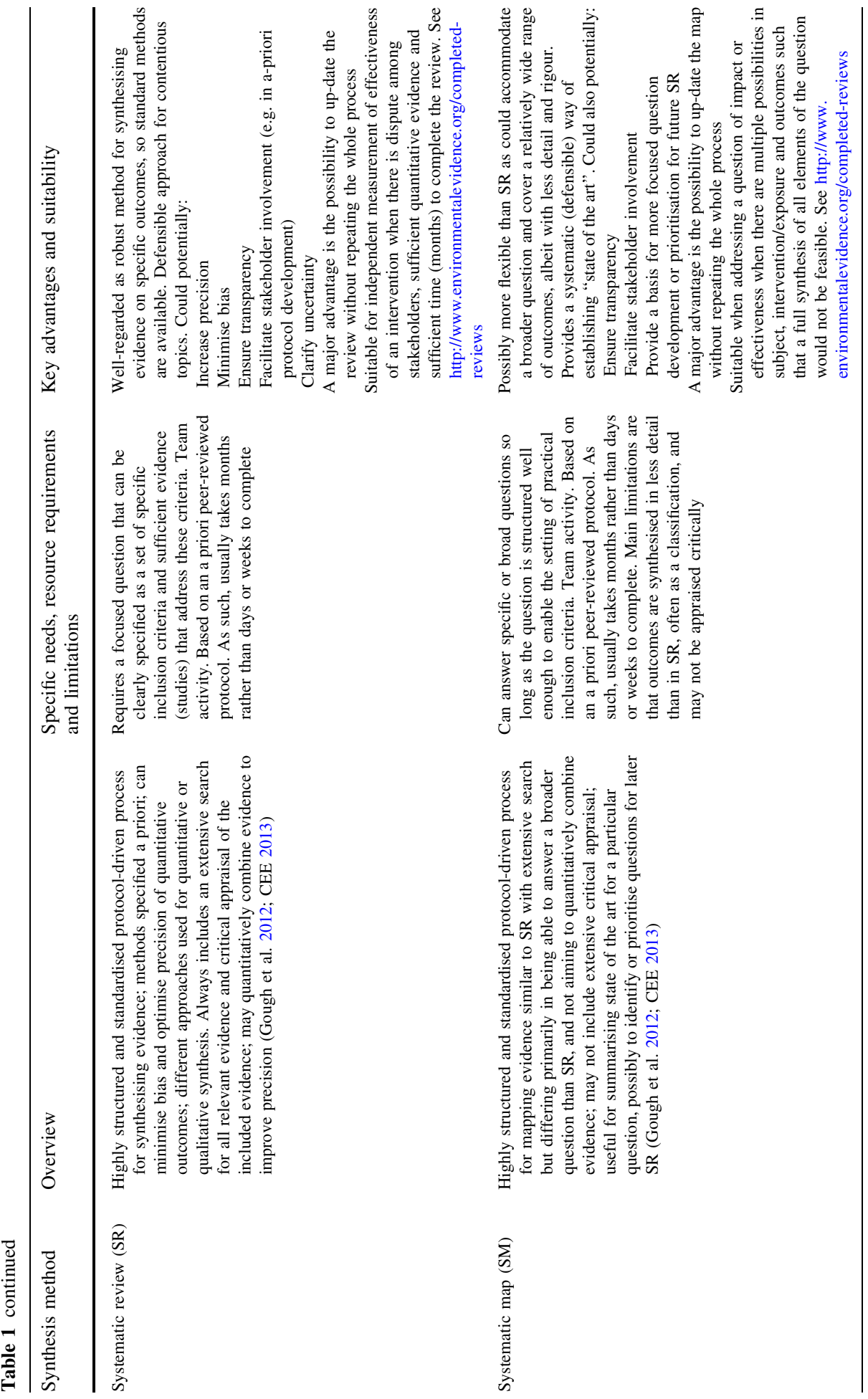




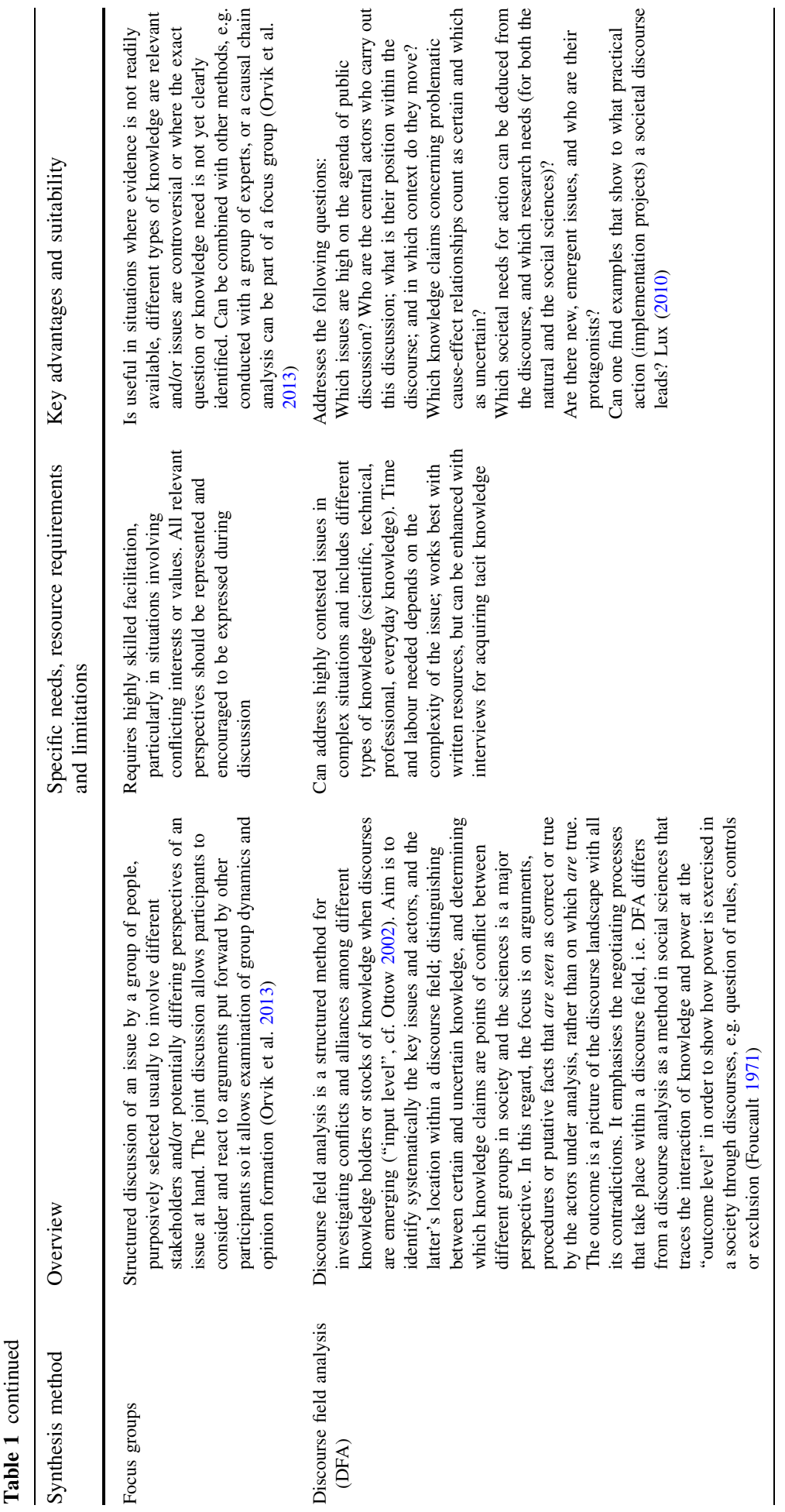




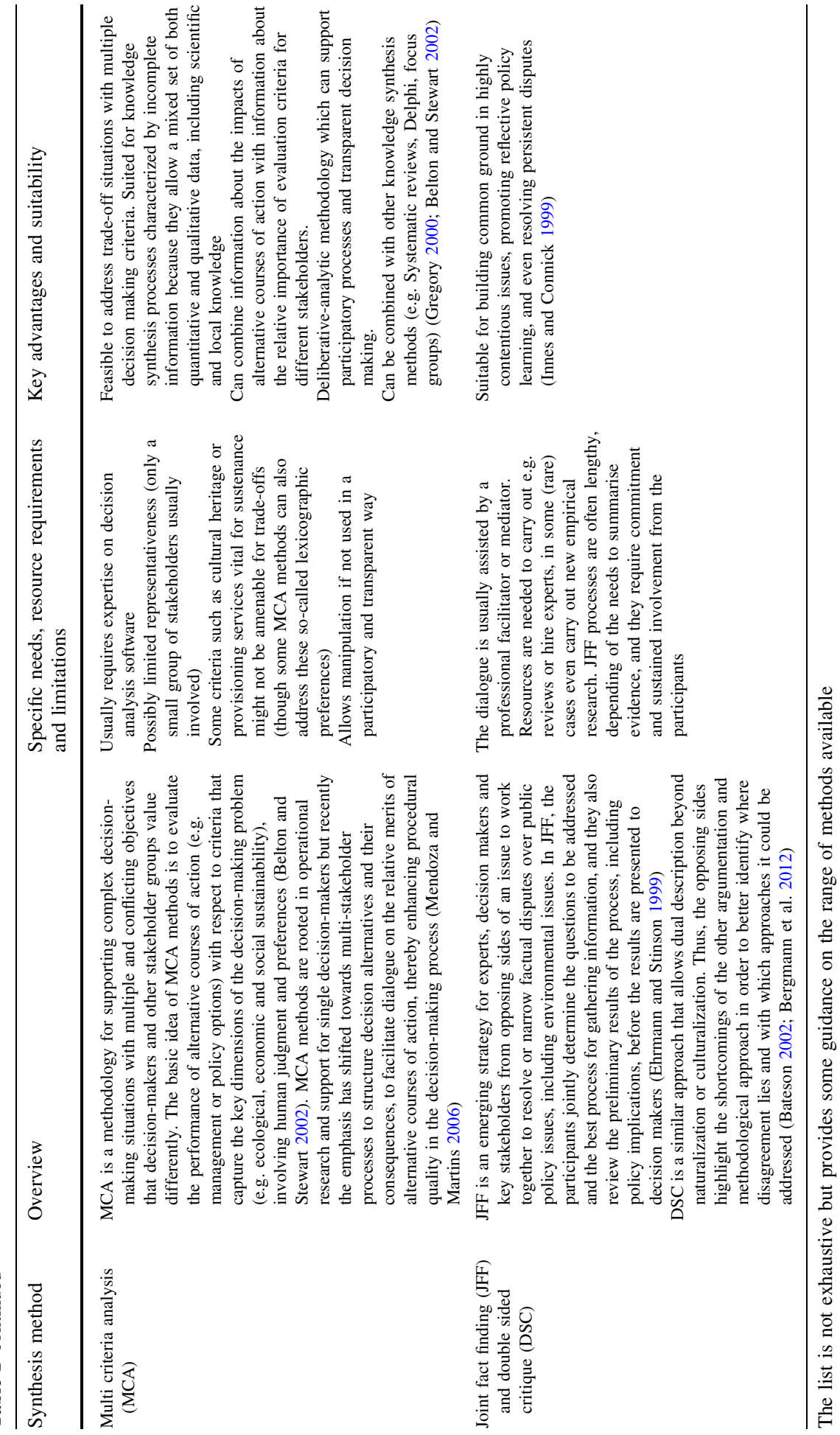




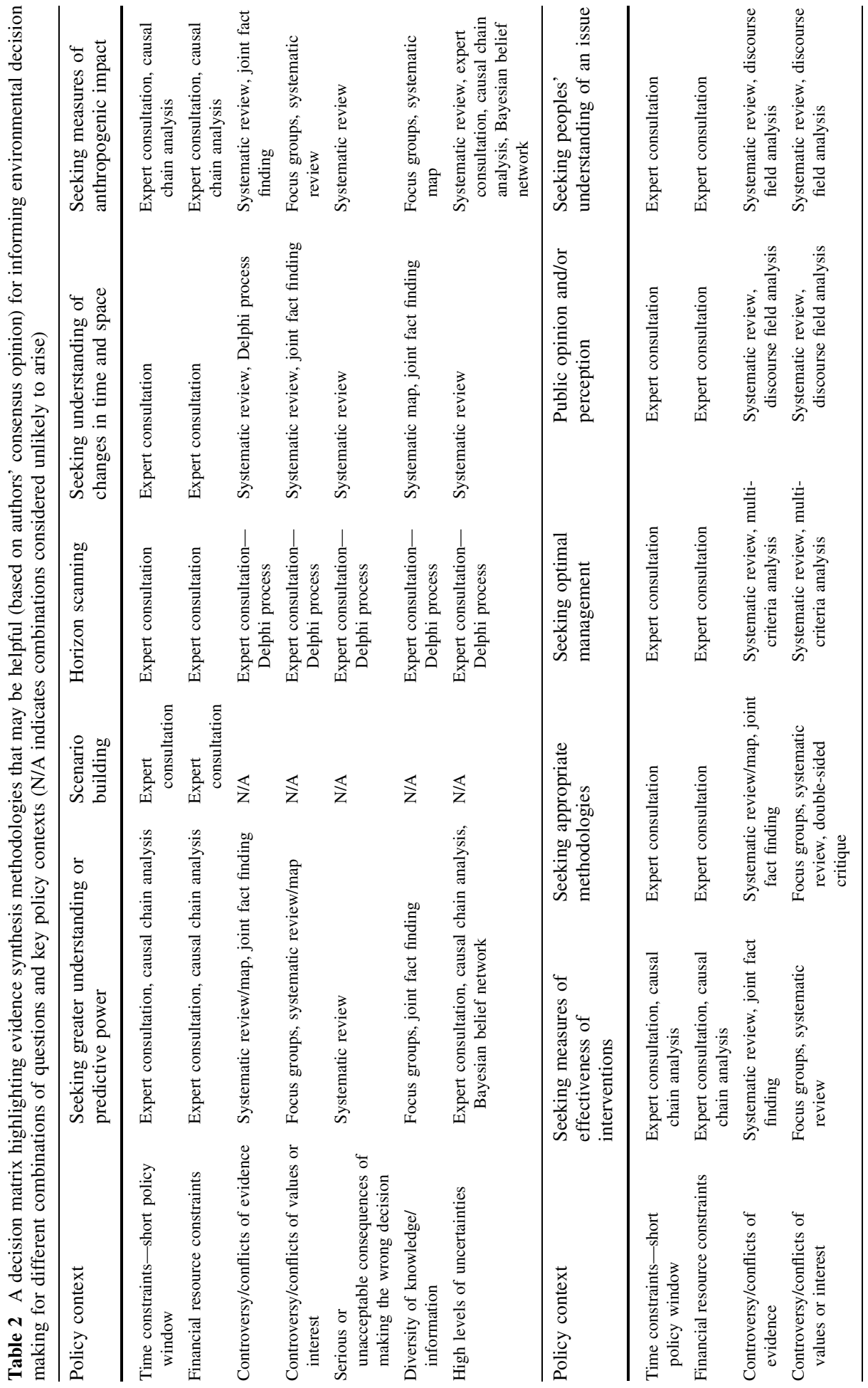




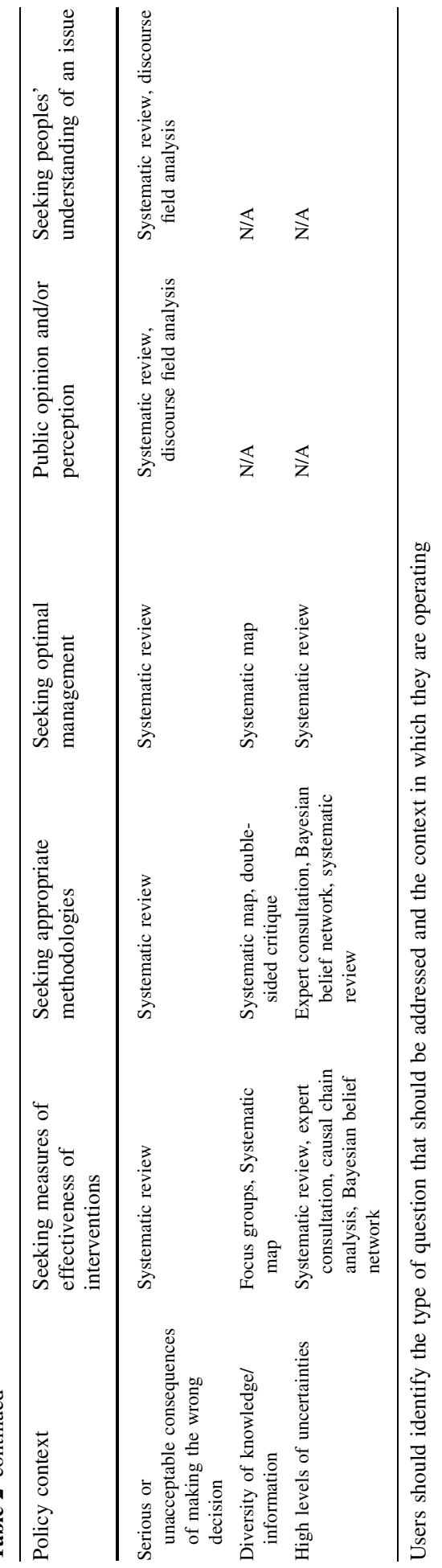




\section{Discussion and conclusions}

The Frankfurt Workshop participants agreed that the diversity of knowledge needs reflected in the range of questions identified by participants in KNEU project workshops requires a diversity of synthesis methodologies. This was confirmed by the formative evaluation of the knowledge synthesis prototype and trial assessments conducted during the KNEU project (Carmen et al. 2015; Schindler et al. 2016).

We recommend the decision matrix (Table 2) suggesting appropriate evidence synthesis methodologies given different types of questions and key policy contexts for use as guidance (not prescription) by those considering commissioning or undertaking a knowledge synthesis to meet their evidence needs and inform their decision making. The classification of questions can help specify what exactly is required to meet knowledge needs and inform policy making at a given stage in policy development or steps in the policy cycle. In the experience of the KNEU project, many of the questions formulated by contributors from the policy community combine several aspects and dimensions and are thus unsuitable for straightforward knowledge synthesis. Hence, a thorough scoping process, in which requesters and experts iteratively negotiate the scope, scale and synthesis methodology, is of paramount importance to maximize quality, credibility and relevance of the output (see Livoreil et al. 2016; Schindler et al. 2016). Similarly, the list of contextual factors can serve as a good starting point to specify the policy context in which the knowledge need arises. Once the type of question is identified clearly and the context specified, the table provides suggestions for which methods might be most useful, though the specificities of each case should be considered (including inter alia what kind of knowledge can be accessed and how, and the level of stakeholder involvement required to resolve potential controversies and conflicts of interest).

The reliability for decision making of the outcomes provided by such methodologies will always depend on how well they are executed. Conforming to the highest standards of these methodologies, including making explicit their potential limitations and built-in biases, is crucial to providing a credible synthesis. There is likely to be a relationship between time and financial constraints and potential reliability of methodology. For example, keeping all other factors (expertise and performance of involved persons) constant, if a quick and low budget synthesis is required and simple expert consultation is employed this is likely to be less reliable than using expert elicitation using the Delphi method that would take longer and cost more (Sutherland and Burgman 2015). A similar comparison could be made between rapid, less structured and less comprehensive literature reviews versus systematic reviews. Furthermore, some problem situations require independently conducted syntheses to reduce susceptibility to bias (Pullin and Stewart 2006) while other situations require participatory, deliberative and reflective inquiry where different interpretive frames and biases engendered in them are critically probed and pitted against each other (Saarikoski 2002, 2007).

In some cases we have identified more than one method and some can be used in combination (e.g. expert consultation and systematic review). In other cases the synthesis method enables further analysis such as cost-benefit evaluation or may enable more accurate modelling of scenarios. These possibilities were considered in the Frankfurt workshop but methods that were not considered to strictly meet our definition of knowledge synthesis were omitted from the table. For example, adaptive management is an approach that might be used for many policy issues (Salafsky et al. 2001), which includes the iterative combination of knowledge synthesis (most often using collaborative methodologies, such as participatory knowledge production and/or multi-criteria analysis; 
e.g. Pahl-Wostl 2007; Méndez et al. 2012) with the generation of new knowledge through the selection, application and monitoring of policies or management strategies (e.g. Walters 1986; Gunderson and Light 2006). It aims at identifying flexible solutions that are resilient to errors and uncertainty (i.e., it treats policies as experiments; Walters and Hilborn 1978; Lee 1993). Hence, while the initial phase of collaborative adaptive management represents a specific type of knowledge synthesis, such an approach extends well beyond the time span of the types of questions addressed here.

In the context of a broader mechanism for biodiversity knowledge synthesis (see Livoreil et al. 2016), the type of matrix shown in Table 2 might be used alongside the scoping process all the way up to agreeing on a methodological protocol. It could help structure the discussion between requesters of a synthesis from the policy community and a knowledge co-ordinating body (i.e. the organisation or individual(s) that would put into place the commissioning and conduct of the synthesis). Such discussions would consider the policy context, the knowledge needs and structure of the question, and would agree on one or more appropriate methodologies.

We recommend that this matrix be further developed, with the inclusion of additional questions and methods in the future. Experiences with using different methods could be documented systematically to start a learning process that might also help to develop more standardized procedures in knowledge synthesis. In the medium term we hope the matrix will stimulate systematic exchange on knowledge synthesis methods and combinations of methods used.

The discussions leading to these results were set in the context of issues involving biodiversity and ecosystem services, but the authors are convinced most of the reasoning outlined above also applies in other policy areas related to, or having an influence on, the environment. Possibly our suggestions might even be helpful regarding knowledge synthesis for decision making in general.

Acknowledgments This work and the workshop it is based on were funded by the KNEU project within the 7th Framework Programme of the European Commission (Contract No.265299).

Open Access This article is distributed under the terms of the Creative Commons Attribution 4.0 International License (http://creativecommons.org/licenses/by/4.0/), which permits unrestricted use, distribution, and reproduction in any medium, provided you give appropriate credit to the original author(s) and the source, provide a link to the Creative Commons license, and indicate if changes were made.

\section{Appendix}

See Table 3.

Table 3 Participants at the Frankfurt workshop. January 13-14th 2014

\begin{tabular}{lll}
\hline 1 & Christoph Görg & UFZ Leipzig, Germany \\
2 & Heidi Wittmer & UFZ Leipzig, Germany \\
3 & Barbara Livoreil & FRB, Paris, France \\
4 & Stefan Schindler & University of Vienna, Austria \\
5 & Marie Vandewalle & UFZ Leipzig, Germany \\
6 & Alexandra Lux & ISOE, Germany \\
\hline
\end{tabular}


Table 3 continued

\begin{tabular}{|c|c|c|}
\hline 7 & György Pataki & $\begin{array}{l}\text { Environmental Social Science } \\
\text { Research Group (ESSRG), Hungary }\end{array}$ \\
\hline 8 & Marie Hubatova & Czech Globe, Czech Republic \\
\hline 9 & Heli Saarikoski & Finnish Environment Institute (SYKE) \\
\hline 10 & Andrew Pullin & Bangor University, UK \\
\hline 11 & Gillian Petrokofsky & Biodiversity Institute, Oxford, UK \\
\hline 12 & Christian Kohl & Julius Kühn-Institut, Germany \\
\hline 13 & Geoff Frampton & $\begin{array}{l}\text { Southampton Health Technology Assessments } \\
\text { Centre (SHTAC),University of Southampton, UK }\end{array}$ \\
\hline 14 & Diana Hummel & ISOE, Germany \\
\hline 15 & Klaus Jacob & FU Berlin, Germany \\
\hline 16 & Aranka Podhora & ZALF, Germany \\
\hline
\end{tabular}

\section{References}

Bateson G (2002) Mind and nature: a necessary unity. Hampton Press, Creskill New edition

Belton V, Stewart TJ (2002) Multiple criteria decision analysis: an integrated approach. Kluwer, London Bergmann M, Jahn T, Knobloch T, Krohn W, Pohl C, Schramm E (2012) Methods for transdisciplinary research. A primer for practice. Campus-verlag, Frankfurt

Breckon J, Dodson J (2016) Using evidence: what works? Alliance for useful evidence. Discussion paper. http://www.alliance4usefulevidence.org/publication/using-evidence-what-works-april-2016/Accessed. Accessed 18 Apr 2016

Carmen E, Nesshöver C, Saarikoski H, Vandewalle M, Watt A, Wittmer H, Young J (2015) Creating a biodiversity science community: experiences from a European Network of Knowledge. Environ Sci Policy 54:497-504

Cash DW et al (2003) Knowledge systems for sustainable development. Proc Natl Acad Sci USA 100:8086-8091

Collaboration for Environmental Evidence (2013) Guidelines for Systematic review and evidence synthesis in environmental management. Version 4.2. http://environmentalevidence.org/wp-content/uploads/ 2014/06/Review-guidelines-version-4.2-final.pdf. Accessed 30 Mar 2016

Department of Environment, Food and Rural Affairs (2015) Emerging tools and techniques to deliver timely and cost effective evidence reviews. Defra Report WT1552. http://sciencesearch.defra.gov.uk/Default. aspx $?$ Menu=Menu\&Module $=$ More $\&$ Location $=$ None $\&$ Completed $=0 \&$ ProjectID $=19265$. Accessed 30 Mar 2016

Dietz T, Stern PC (1998) Science, values, and biodiversity. Bioscience 48:441-444

Ehrmann J, Stinson B (1999) Joint fact finding and the use of technical experts. In: Susskind L, McKearnan $\mathrm{S}$, Thomas-Larmer $\mathrm{J}$ (eds) The consensus building handbook. A comprehensive guide to reaching agreement. Sage Publications, Thousand Oaks, pp 375-398

Fenton NE, Neil M (2012) Risk assessment with Bayesian networks. CRC Press, Boca Raton

Foucault M (1971) Orders of discourse. Soc Sci Inf 10:7-30

Gough D, Oliver S, Thomas J (2012) An introduction to systematic reviews. Sage, London

Gregory R (2000) Valuing environmental policy options: a case study comparison of multiattribute and contingent valuation survey methods. Land Econ 76(2):151-173

Gunderson LH, Light SS (2006) Adaptive management and adaptive governance in the Everglades ecosystem. Policy Sci 39:323-334

Innes J, Connick S (1999) San Fransisco estuary project. In: Susskind L, McKearnan S, Thomas-Larmer J (eds) The consensus building handbook: a comprehensive guide to reaching agreement. Sage, Thousand Oaks, pp 801-827

Jordan A, Russel D (2014) Embedding the concept of ecosystem services? The utilisation of ecological knowledge in different policy venues. Environ Plan C Gov Policy 32:192-207

Lee KN (1993) Compass and gyroscope: integrating science and politics in the environment. Island Press, Washington D.C.

Linstone HA, Turoff M (eds) (2002) Delphi method: techniques and applications. Addison-Wesley Pub. Co., Advanced Book Program, Reading 
Livoreil B, Geijzendorffer I, Pullin AS, Schindler S, Vandewalle M, Nesshöver C (2016) Biodiversity knowledge synthesis at the European scale: actors and steps. Biodivers Conserv. (this issue)

Lux A (2010) Updates: current developments in the discourse field. biodiversity \& climate 2. Knowledge Flow Paper Nr. 7. Frankfurt am Main: http://www.isoe.de/ftp/bik_f/kfp_nr-7.pdf. Accessed 17 Apr 2016

Méndez PF, Isendahl N, Amezaga JM, Santamaría L (2012) Facilitating transitional processes in rigid institutional regimes for water management and wetland conservation: experience from the Guadalquivir Estuary. Ecol Soc 17:26

Mendoza GA, Martins H (2006) Multi-criteria decision analysis in natural resource management: a critical review of methods and new modelling paradigms. For Ecol Manag 230:1-22

Mukherjee N, Hugé J, Sutherland WJ, McNeill J, Van Opstal M, Dahdouh-Guebas F, Koedam N (2015) The Delphi technique in ecology and biological conservation: applications and guidelines. Methods Ecol Evol 6:1097-1109

Nesshöver C et al (2016) The network of knowledge approach-improving the science and society dialogue on biodiversity and ecosystem services in Europe. Biodivers Conserv. (this issue)

Orvik A, Larun L, Berland A, Ringsberg KC (2013) Situational factors in focus group studies: a systematic review. Int J Qual Methods 12:338-358

Ottow R (2002) Ein Modell politischer Diskursanalyse. Eur J Sociol 43:241-269

Owens S (2005) Making a Difference? Some perspectives on environmental research and policy. Trans Inst Br Geogr New Ser 30:287-292

Pahl-Wostl C (2007) Transitions towards adaptive management of water facing climate and global change. Water Resour Manag 21:49-62

Pullin AS, Stewart GB (2006) Guidelines for systematic review in conservation and environmental management. Conserv Biol 20:1647-1656

Saarikoski H (2002) Naturalized epistemology and the dilemmas of planning practice. J Plan Educ Res 22:3-14

Saarikoski H (2007) Objectivity and the environment-epistemic value of biases. Environ Polit 16:488-498

Salafsky N, Margoulis R, Redford K (2001) Adaptive management-a tool for conservation practitioners. Biodiversity Support Program, Washington D.C.

Sarkki S, Niemela J, Tinch R, Van den Hove S, Watt A, Young J (2013) Balancing credibility, relevance and legitimacy: a critical assessment of trade-offs in science-policy interfaces. Sci Public Policy 42:676-689

Schindler S, Livoreil B, Sousa Pinto I, Araujo R, Zulka KP, Pullin AS, Santamaria L, Kropik M, FernandezMendez P, Wrbka T (2016) The network biodiversity knowledge in practice: insights from three trial assessments. Biodivers Conserv. (this issue)

Sharman A, Holmes J (2010) Evidence-based policy or policy-based evidence gathering? Biofuels, the EU and the $10 \%$ target. Environ Policy Gov 20:309-321

Sutherland WJ, Burgman M (2015) Policy advice: use experts wisely. Nature 526:317-318

Sutherland WJ, Armstrong-Brown S, Armsworth PR et al (2006) The identification of one hundred ecological questions of high policy relevance in the UK. J Appl Ecol 43:617-627

Uusitalo L (2007) Advantages and challenges of Bayesian networks in environmental modelling. Ecol Model 203:312-318

Walters C (1986) Adaptive management of renewable resources. Blackburn Press, Caldwell

Walters CJ, Hilborn R (1978) Ecological optimisation and adaptive management. Annu Rev Ecol Syst 9:157-188 